\title{
Dynamic measurement of the helium concentration of evolving tungsten nanostructures using Elastic Recoil Detection during plasma exposure
}

\author{
K.B. Woller*, D.G. Whyte, G.M. Wright \\ MIT Plasma Science and Fusion Center MIT, 175 Albany St., Cambridge, MA, USA, 02139
}

\begin{abstract}
:
Helium (He) concentration depth profiles of evolving tungsten (W) nanostructures have been measured for the first time using in situ Elastic Recoil Detection (ERD) throughout plasma irradiation. Exposures resulting in fuzzy and non-fuzzy surfaces were analyzed in order to illuminate the role of He during the development of these surface morphologies. ERD was performed on samples with surface temperatures from $\mathrm{T}_{\mathrm{s}}=530-1100 \mathrm{~K}$ and irradiated by $\mathrm{He}$ flux densities of $\Gamma_{\mathrm{He}} \sim 10^{20}-10^{22} \mathrm{~m}^{-2} \mathrm{~s}^{-1}$. He concentration profiles in samples that developed either non-fuzzy or fuzzy surfaces are uniformly shaped with concentrations of 1.5 to 7 at.\%, which is presumed to be too low for pressure driven growth models. Therefore, surface morphology changes are not perpetuated by continuous bubble bursting deformation. Also, a threshold in He flux density above $10^{20} \mathrm{~m}^{-2} \mathrm{~s}^{-1}$ is suggested by using in situ ERD to monitor the depth profile evolution of the He-rich layer while changing the flux during exposure.

PACS: 52.40.Hf, 82.80.Yc, 81.07-b, 81.05.Je

PSI-21 keywords: W fuzz, Helium, Surface analysis, Bubbles \& Blisters, Divertor material *Corresponding author address: MIT Plasma Science and Fusion Center, 175 Albany St., NW17-210, Cambridge, MA, USA, 02139

*Corresponding author e-mail: kbwoller@mit.edu

Presenting author: Kevin B. Woller
\end{abstract}




\section{Introduction}

Tungsten (W) nanostructures are of interest in many areas of materials research due to their drastic effects on the properties of the W surface. Arborescent nanostructures first occurred unexpectedly [1] and thus became a topic of alarm and intrigue because of their development under similar conditions as are expected in the ITER divertor and DEMO walls. Despite extensive study of the surface changes and performance, we still have limited understanding of how W nano-tendrils, or "fuzz," grow. If W fuzz is a desired surface modification from an engineering point-of-view, understanding the growth mechanisms might serve to optimize and accelerate specific W fuzz development, or, if not, suppress it. There are two basic premises for modelling $\mathrm{W}$ fuzz growth: pressure driven deformation [2] and surface diffusion around deformities $[3,4,5]$. If bubbles were to burst to deform the surface, pressure would have to build large enough to overcome the yield strength of W. However, many experiments performed on $\mathrm{W}$ fuzz after growth have shown that the He bubbles do not retain these required high pressures once the plasma exposure is ceased [6, 7]. In this work, we measure the He concentration during fuzz development to show that the He concentration, even during growth, is less than what is thought necessary for bubble bursting models. In situ ERD has been developed and performed on samples grown under various conditions within the growth parameter space for W fuzz. First, an overview of the measurement technique and considerations for implementation near such an extreme environment are given. Then, the $\mathrm{He}$ concentrations as well as dynamic parameters are presented and discussed.

\section{Materials and Methods}

\subsection{Simultaneous ERD and RBS setup}

He concentration measurements were carried out using the Dynamics of ION Implantation and Sputtering Of Surfaces (DIONISOS) experiment [8]. Fig. 1 shows a top-down schematic 
of the measurement set up. Essentially, DIONISOS has the capabilities of ordinary Ion Beam Analysis (IBA) techniques with the added ability to irradiate the sample at the same time.

A $7 \mathrm{MeV}$ oxygen-16 (O) ion beam was directed at an angle of 75 degrees normal to the surface of the samples. A $1 \mathrm{~mm}$ wide slit aperture in front of the detector defined the nominal scattering angle of 30 degrees from the incident beam path. The solid angle subtended by the detector aperture was measured by performing ERD on a Kapton target while monitoring the beam current periodically with a faraday cup. The yield of the hydrogen $(\mathrm{H})$ from the Kapton was simulated using SimNRA [9]. The solid angle determined in this manner was $3.22+/-0.1$ msr. The thickness and roughness of Diamond-like Carbon (DLC) foils acquired from MICROMATTER ${ }^{\mathrm{TM}}$ measured using Rutherford Backscattering Spectroscopy (RBS) were $5012 \times 10^{15}$ atoms $/ \mathrm{cm}^{2}$ and $989 \times 10^{15}$ atoms $/ \mathrm{cm}^{2}$, respectively, using SimNRA to fit the foil spectrum. To overcome the bane of scattered ion beam leakage through stopper foil pinholes, a stack of 9 of these DLC foils was placed after the aperture to stop the O scattered off of W from reaching the detector. An Ortec ULTRA charged particle detector was positioned behind the stopping foil stack to measure the final energy of the recoiled He. The recoiled energy scale of the detection set up was calibrated using separate proton and alpha ion beams of various known energies scattered off of a quartz target at a scattering angle of 45 degrees. The overall depth resolution of the detection set up during irradiation was determined from this calibration and by an electronic pulser signal supplied to the detector preamplifier. The depth resolution was approximately $6 \mathrm{~nm}$ for He at the surface of W with a bulk density, or $40 \mathrm{x}$ $10^{15} \mathrm{~W}$ atoms $/ \mathrm{cm}^{2}$.

The probing beam current, at approximately $1 \mu \mathrm{A}$, could not be measured directly during irradiation due to the several hundred $\mathrm{mA}$ of ion saturation current supplied to the sample by the plasma. Thus, the beam dose was calculated by measuring the RBS yield of $\mathrm{O}$ particles scattered from $\mathrm{W}$ at an angle of 90 degrees from the incident beam path with a second 
detector simultaneous with the ERD detector. A single DLC foil carefully selected to minimize any pinholes was used in front of this detector to make it light-tight during plasma operation. The solid angle of the aperture in front of this detector was calibrated prior to irradiation experiments by measuring the beam current to a $\mathrm{W}$ sample that had secondary electrons suppressed. As before, the yield from this RBS signal was fit using SimNRA and the solid angle was measured to be $9.6+/-0.1 \times 10^{-6} \mathrm{sr}$.

\subsection{In situ ERD and RBS Considerations}

Now, we present considerations taken to implement these in situ IBA techniques in such a dynamic environment. Particle trajectories deviate from the linear geometry of the measurement set up due to the magnetic field used for the plasma source and confinement. The magnetic field that the whole experiment is bathed in is very uniform and ranges from $230-675 \mathrm{G}$ depending on the desired plasma mode. The trajectory deviations were calculated using the Lorentz force in a uniform magnetic field to be near 1 degree and were taken into account during the data analysis in SimNRA. This geometrical change results in a slight shift in the detected energy scale. However, the shift is much smaller than the detection resolution and does not result in any significant source of error.

The background neutral He gas in the chamber, with a pressure of 3-4 Pa, also interacts with the probing $\mathrm{O}$ beam creating an artifact at higher recoiled energies due to the lower recoil angle [8]. However, in this case, this background signal does not interfere with the He signal from the $\mathrm{W}$ surface because the geometry of the reaction requires He particles detected with the surface recoil energy or less to come from the sample. There is also a low energy background signal that is constant throughout the exposure. As Hydrogen $(\mathrm{H})$ has long thermal desorption times even at these temperatures [10], the low energy background is likely due to multiple scattering of $\mathrm{H}$ and thus was subtracted away when necessary. These artifacts are discussed further below. 
The ERD detector was in close proximity to an actively heated sample and a radiofrequency $(\mathrm{RF})$ generated plasma column. If the detectors are not maintained at room temperature or below, then the resolution of the measurement is degraded. Also, the RF plasma was found to cause electromagnetic interference (EMI) in the ERD detector signal. To maintain low leakage current in the solid state detectors, the detectors were housed in watercooled housing. In the most extreme case, the leakage current in the ERD detector increased by a factor of 2 indicating that the detector temperature increase was only about $5{ }^{\circ} \mathrm{C}$. The decrease in resolution was monitored by a pulser signal supplied to the detector preamplifier during irradiation measurements and the previously mentioned depth resolution was taken as the predominant case. The detectors were also insulated from the detector holders so that any EM waves generated by the plasma would be grounded to the vacuum vessel promptly without influencing the detector signal.

\subsection{Sample Growth Conditions}

The $\mathrm{W}$ used for these measurements were $25.4 \mathrm{~mm}$ diameter and $1.5 \mathrm{~mm}$ thick PLANSEE 99.95 weight $\%$ tungsten coins. They were mechanically polished to a mirror finish. Samples were then either actively heated with a HeatWave Labs UHV heater or mounted on a water cooled target holder. The heater controller uses a type K thermocouple in contact with the alumina base of the heater stage just $1 \mathrm{~mm}$ from the sample for feedback. It has been observed that the emissivity of W surfaces increases with the development of fuzz [11]. Since the heater had feedback independent of the sample, the sample surface temperature is more easily regulated by the stage and not by the plasma heat flux. Thus, an increase in the emissivity due to nano-tendril development results in increased heat loss due to radiation and decreased plasma heat conduction to the sample, but this is compensated for by the stage, holding the

temperature nearly constant. The samples without active feedback for temperature control did not develop fuzz. The surface temperatures of these samples were monitored using an infrared 
(IR) camera responsive to a wavelength of $1.6 \mu \mathrm{m}$ using an emissivity of $\varepsilon=0.34$ as determined by a separate temperature calibration on a polished $\mathrm{W}$ sample with a thermocouple spot-welded to its surface at $1020 \mathrm{~K}$.

The IR camera was not available for all irradiation experiments. A slab model of the sample during exposure was used to calculate the surface temperature given the heat flux from the ion beam, plasma, and heater and the heat lost due to radiation. Conduction through the sample holding clips was neglected. This model adequately reproduced the surface temperature as measured by the IR camera when it was available both before irradiation and after the plasma was ceased when changes in emissivity were indicated and thus served to accurately calculate the surface temperature when it was unavailable. The surface temperatures of Table 1. are the results from this surface temperature calculation.

A helicon plasma source was used to generate plasmas with various He ion flux densities from $2.4 \times 1020 \mathrm{~m}-2 \mathrm{~s}-1$ to $1.6 \times 1022 \mathrm{~m}-2 \mathrm{~s}-1$ where the plasma parameters were measured using electrostatic double probes. During irradiation, the samples were biased to $-50 \mathrm{~V}$ with respect to the vacuum vessel to accelerate the irradiating plasma He ions to an energy of $\sim 60$ $\mathrm{eV}$ assuming the plasma potential is $\sim 3 \mathrm{k}_{\mathrm{B}} \mathrm{T}_{\mathrm{e}}$ and the plasma is mostly singly ionized He. The plasma composition was not measured directly, but the samples were evaluated with Energy

Dispersive X-ray spectroscopy (EDS) using a Bruker EDS system on a JEOL 5910 Scanning Electron Microsope (SEM). The concentrations of common impurity species carbon (C) and oxygen $(\mathrm{O})$ left in the irradiated samples were the same as for a non-irradiated sample indicating either a negligible amount of these impurities in the plasma or the net deposition of these impurities was imperceptible.

\subsection{ERD Spectra Analysis}

ERD spectra were simulated using SimNRA [9]. Using the known details of the experiment, the $\mathrm{W}$ and He content (presented as areal densities) of the He-rich layer were fit 
to the simulation of each spectrum and the results compiled to show the time evolution of these measurements. Fig. 2 shows the analysis process from one time slice of sample B near the end of the irradiation. The top plot shows the raw ERD spectrum acquired after a long time into the irradiation (boxes) along with the background signal measured before the irradiation was initiated (exes). In the background spectrum, at low channel number, is a persistent signal that appears when the beam is incident on the $\mathrm{W}$ sample. A possible cause is as follows. Sample B has been heated to $1050 \mathrm{~K}$, so the entire surface $\mathrm{H}$ has been desorbed at the point when the background signal was measured. The low channel background occurs around the same channel numbers as we have calibrated the detection system for the surface $\mathrm{H}$ signal. Recoils generated from the surface of materials would be at higher channel numbers than from the bulk. Therefore, we believe the low channel background could be H outgassing from the bulk W as we stated above in section 2.2. In the mid-channel number range of the background plot is the artifact associated with the background neutral gas pressure. This background signal was measured when a He gas feed lead to $3.8 \mathrm{~Pa}$ of gas pressure in the vacuum vessel. If the sample were removed completely so that the beam could traverse the entire vacuum vessel while filled with low pressure He, this artifact stretches from where it appears in Fig. 2 down to channel 0. This confirms that with the $\mathrm{W}$ sample in place, the beam is blocked from forward recoiling any more He gas towards the detector and the artifact of the background signal does not contribute to the He signal measured during irradiation. Also present in the back ground signal at high channel numbers is a pulser signal we generate to monitor the performance of the detection system. All of these background components are constant before, during, and after irradiation, so they can be discarded with confidence.

The lower plot of Fig. 2 shows the raw ERD signal with the background removed along with the SimNRA simulated spectrum used to fit the He content and W areal density within the He-containing layer on the surface. Since the He is so uniformly distributed throughout 
the He-containing layer, the average He concentration in that layer is simply the ratio of the He content to the total content in that layer. Also, at the outset of the irradiation time, the entire He signal is isolated from any other signal interference. As indicated in [12], if the entire recoil spectrum is captured then the full content of that layer is directly measured whether the surface is rough or not. For our measurements, we present the He content for as long as we capture the entire recoil signal. As soon as the layer is too thick, we cannot report on the changes in the profile any longer with this same level of certainty. Thus, the He concentration evolution we present here is independent of the surface morphological evolution.

\section{Results and Discussion}

Table 1 summarizes the various growth conditions implemented in this work. Once the exposures were completed, the surface morphologies were imaged using the Helios NanoFab SEM of the MIT CMSE Shared Experimental Facility. Images of the final surface morphologies are shown in Fig. 3. Sample C was exposed to two different plasmas sequentially during its exposure. At first, sample $\mathrm{C}$ was exposed to a flux density similar to sample E, but with a higher surface temperature of $1087 \mathrm{~K}$ in hopes of reaching a $\mathrm{W}$ fuzz growth regime. However, after some time, sample $\mathrm{C}$ was showing He saturation in a thin layer at the surface of the W. So, the He flux density was increased and then sample C showed signs of morphology changes through the active ERD spectra. This is illustrated in Fig. 3 through the evolution of the $\mathrm{W}$ areal density and He content, respectively, in the Herich layer of sample $\mathrm{C}$ with the open and closed diamond symbols. $\mathrm{W}$ areal density is used as a measure of the "thickness" of the He-containing layer because the porosity of this layer is changing during exposure. The $\mathrm{W}$ areal density is independent of the porosity, because we are no longer confining ourselves to linear dimensions. Thus, comparison of the evolution of the $\mathrm{W}$ areal density within the developing He layer between separate irradiation experiments may 
be done despite the surface morphological evolution. With the first flux density on sample C, the thickness approaches a value near $200 \times 10^{15} \mathrm{~W} / \mathrm{cm}^{2}$, similar to samples E, F, and G, which did not produce $\mathrm{W}$ fuzz. In bulk $\mathrm{W}$, this corresponds to $\sim 30 \mathrm{~nm}$. This thickness for a He bubble containing layer in $\mathrm{W}$ is on the order of what others have seen for $\mathrm{W}$ that did not produce fuzz [13]. Each sample was exposed to He ions of the same energy and that might be why samples E, F, and G equilibrate to the same depth. After increasing the He flux density, the thickness increases at a rate similar to samples A, B, and D, which grew W fuzz.

There is also a remarkable difference between the outcomes of samples D and E. These samples were exposed with the same temperature, but with vastly different flux densities. Also, the surface modifications are vastly different where in sample D developed fuzz readily and sample $\mathrm{E}$ was quite bare. This may indicate a flux density threshold that factors into the growth parameter space of $\mathrm{W}$ fuzz. However, there is also a factor of 10 difference between the final He fluences to the samples, with sample E supplied with less fluence than sample D. This difference in exposure may also account for the stark differences in surface morphology.

The top of Fig. 4 shows the He concentration evolution for each of the samples. During the first 400 s or so, the He concentration increases. Then, the He concentration reaches a saturation level. For samples A, B, and D, at the same time as saturation, the surface reflectivity appeared to decrease, as observed by eye, suggesting W fuzz had begun to grow.

Over the $\mathrm{W}$ fuzz growth parameter range of this study, the He concentration saturates to a value between 1.5 and 7 at. \%. This He concentration range is in agreement with post exposure measurements made previously on other samples produced with various linear plasma devices [6]. The value of this saturation level and time to saturation are dependent on the growth conditions. The He concentration appears to be monotonic with surface temperature. This is opposite to hydrogenic retention, where retention decreases rapidly with temperature. This demonstrates nicely that there are fundamental differences between H/D/T 
trapping and He trapping (i.e. vacancy trapping vs. bubble filling). Previous ex situ measurements did not show such a clear relationship with surface temperature.

After the plasma exposure was ceased, these He concentration levels remained in the samples. This means that the He measured during exposure is truly that which is permanently trapped. Thus, there is no significant contribution of solute interstitial He in $\mathrm{W}$ observed in the measurements. Also, the amount of $\mathrm{He}$ in the space between the nano-tendrils is negligible, as it should be. As was suggested with ex situ measurements [6], the He concentration measured is inconsistent with speculation of void creation by loop-punching He bubbles. The presence of high pressure He certainly provides stresses to sustain W fuzz development. Also, the bubbles deform the surface, creating trapping sites for $\mathrm{W}$ adatoms [4]. In the low temperature regime, the $\mathrm{W}$ does not readily move around the He bubbles so fuzz is not created. As the surface temperature is increased, the $\mathrm{W}$ congregates to the bubble apexes and ridges where new bubbles can form and the process repeated [3]. The bubble supply rate has to be adequate for fuzz to develop, as was observed with sample $\mathrm{C}$. This shows that there is a He flux density threshold for W fuzz growth as well.

\section{Conclusions}

Elastic Recoil Detection (ERD) has been used for the first time to measure the dynamic He concentration in $\mathrm{W}$ during plasma irradiation. The He concentrations of various samples were measured to be in the range of 1.5-7 at. \%. These concentrations are not adequate to support loop-punching as a fuzz growth perpetuation mechanism [2]. The individual He concentration depth profiles of fully developed W fuzz layers show the He concentration to be uniform through the measurable "depth," which varies with the porosity of the He containing layer.

He agglomeration and elevated surface temperature are the critical ingredients in the process that results in $\mathrm{W}$ fuzz from He irradiation [14]. Current theories and modeling suggest 
that the nucleation of high pressure, high density He bubbles at trapping sites progress into clusters, deforming the $\mathrm{W}$ surface and providing the initial deformation that leads to tendril growth $[5,15]$. Our results introduce a constraint on modeling this process, namely, that the overall He concentration of evolving layers both during and after He irradiation remains in the single at. \% range.

\section{Acknowledgements}

This work is supported by US DOE award DE-SC00-02060. This work made use of the MRSEC Shared Experimental Facilities at MIT, supported by the National Science Foundation under award number DMR-08-19762.

\section{References}

[1] S. Takamura, et al., Plasma Fusion Res. 1 (2006) 051.

[2] G.M.Wright, et al., J. Nucl. Mat. 438 (2013) S84.

[3] S.I. Krasheninnikov, et al., Nucl. Fusion 54 (2014) 073019.

[4] Y.V. Martynenko, M.Y. Nagel, Fizika Plazmy 38 (2012) 1082.

[5] S. Kajita, et al., Nucl. Fusion 49 (2009) 095005.

[6] K.B. Woller, et al., J. Nucl. Mat. 438 (2013) S913.

[7] S. Kajita, et al., J. Nucl. Mat. 421 (2012) 22.

[8] G.M. Wright, et al., Rev. Sci. Instrum. 85 (2014) 023503.

[9] M. Mayer, SIMNRA User's Guide, Report IPP 9/113, Max-Planck-Institut für

[10] H. Maier, et al., This issue.

[11] S. Takamura, et al., J. Nucl. Mat. 438 (2013) S814. Plasmaphysik, Garching, Germany, 1997.

[12] R. Behrisch, et al., Nucl. Instrum. Meth. B, 136-138 (1998) 628-637.

[13] M. Miyamoto, et al., J. Nucl. Mat. 415 (2011) S657.

[14] M.J. Baldwin, R.P. Doerner, Nucl. Fusion 48 (2008) 035001. 
[15] F. Sefta, et al., Nucl. Fusion 53 (2013) 073015. 
Figure captions:

Fig. 1. Schematic looking down on the ion beam analysis set up within the DIONISOS irradiation chamber. The probing ion beam species was ${ }^{16} \mathrm{O}$ accelerated to $7 \mathrm{MeV}$ by a tandem electrostatic accelerator. The sample was tilted $30^{\circ}$ with respect to the magnetic field and $75^{\circ}$ from the probing beam. A detector at $30^{\circ}$ from the incident probing beam path collected recoiled species from the surface of the irradiated samples. A detector at a scattering angle of $90^{\circ}$ collected scattered $\mathrm{O}$ from the surface as a measure of the beam dose.

Table 1. Sample growth conditions. $\mathrm{E}_{\mathrm{He}}=60 \mathrm{eV}$.

Fig. 2. Above, raw ERD spectra of a time slice of sample b after $3000 \mathrm{~s}$ of irradiation (filled boxes) and the background signal measured before irradiation had begun, but with the sample at $1050 \mathrm{~K}$ and a background $\mathrm{He}$ gas pressure of $3.8 \mathrm{~Pa}$ (exes), note the log scale so that the $\mathrm{He}$ gas artifact may be seen around channel 800 . Below, the same time slice of sample b with the background signal removed (dotted line) along with the simulated spectrum from SimNRA (solid line), note the linear scale.

Fig. 3. SEM micrographs of the final surface morphologies grown under various surface temperatures, He flux densities, and He fluences in DIONISOS as indicated in Table 1.

Fig. 4. Results from samples that eventually developed fuzzy surfaces are on the left and results from samples that did not develop fuzzy surfaces are on the right. The He concentration evolution is on top, He content evolution is in the middle, and, on bottom, the $\mathrm{W}$ areal density evolution of He-rich layers in $\mathrm{W}$ irradiated with He under the conditions of Table 1. Error bars were excluded for clarity. The error for the He concentration is approximately $15 \%$ mainly because the error in the He content is up to $15 \%$ as well. The error for the $\mathrm{W}$ areal density is typically $3 \%$. 
Fig. 1

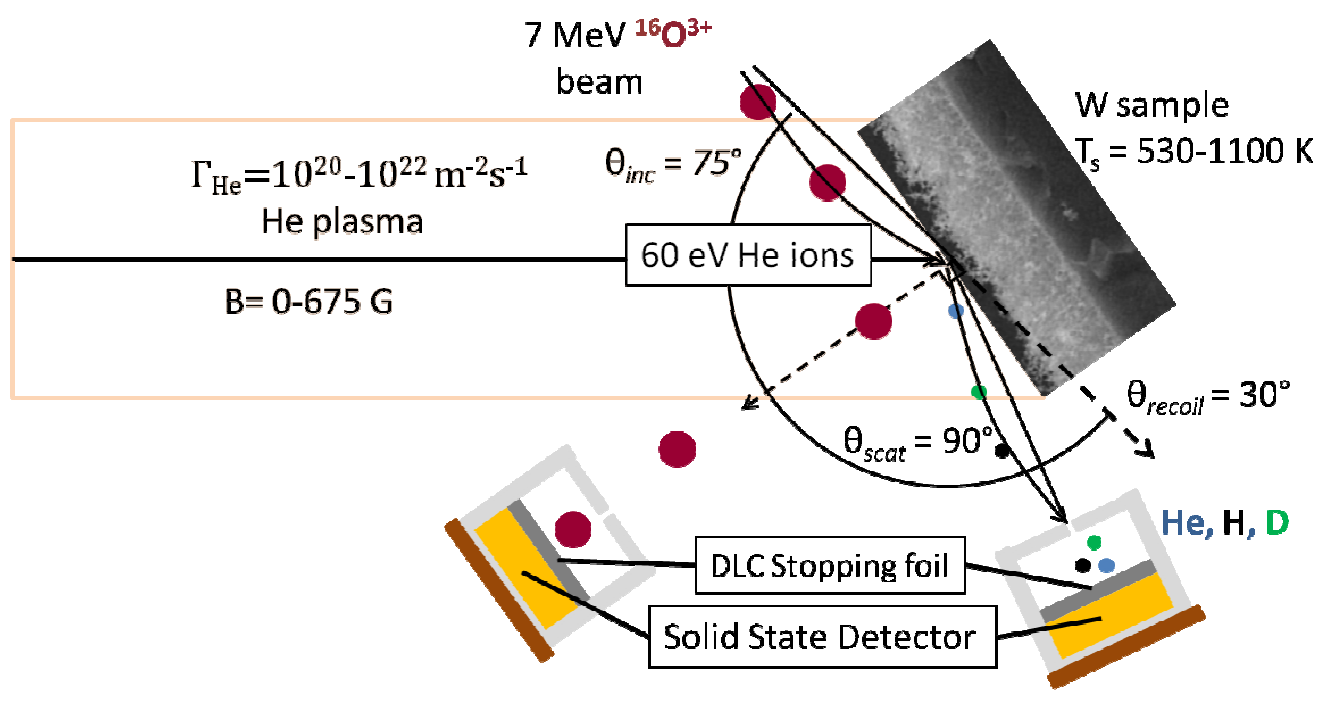


Table 1.

\begin{tabular}{lccc}
\hline Sample & $T_{s}, K$ & $\Gamma_{H e}, 10^{20} \mathrm{~m}^{-2} \mathrm{~s}^{-1}$ & $\Phi_{H e}, 10^{23} \mathrm{~m}^{-2}$ \\
\hline $\mathrm{a}$ & 1050 & 160 & 803 \\
$\mathrm{~b}$ & 1043 & 100 & 629 \\
High flux c & 1107 & 100 & 415 \\
$\mathrm{~d}$ & 1103 & 56 & 49 \\
Low flux c & 1087 & 2.5 & -- \\
$\mathrm{e}$ & 1057 & 2.5 & 2.6 \\
$\mathrm{f}$ & 874 & 44 & 40 \\
$\mathrm{~g}$ & 527 & 56 & 60 \\
\hline
\end{tabular}


Fig. 2

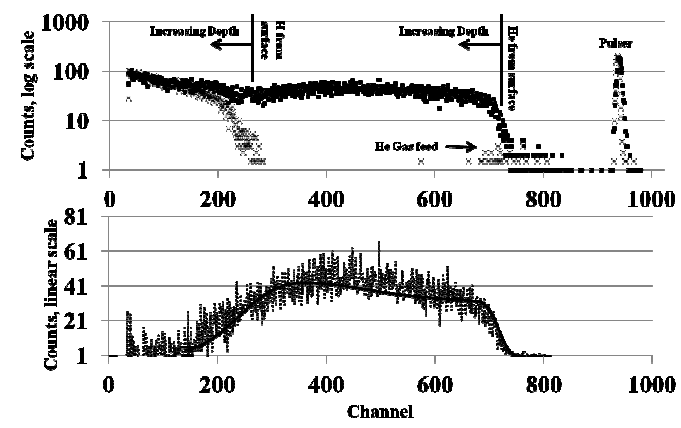


Fig. 3

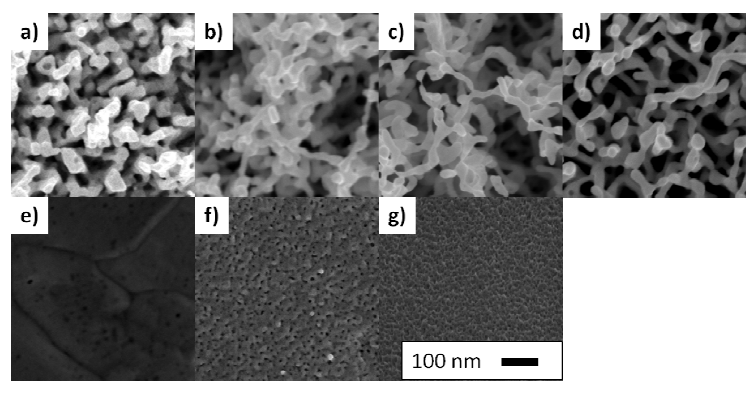


Fig. 4
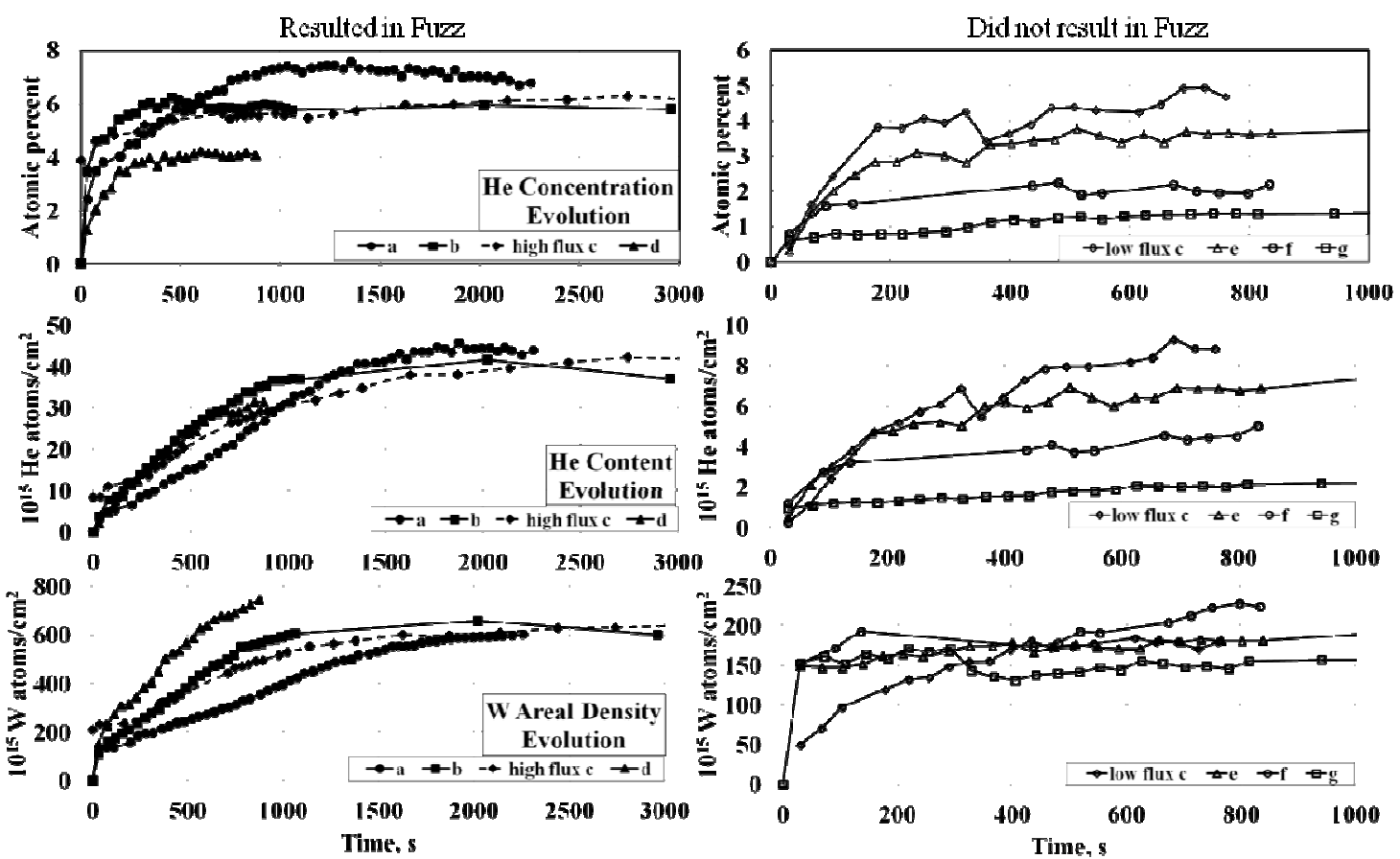